\title{
Using an e-Delphi technique in achieving consensus across disciplines for developing best practice in day surgery in I reland
}

\author{
Babak Meshkat ${ }^{1}$, Seamus Cowman ${ }^{2}$, Georgina Gethin ${ }^{2}$, Kiaran Ryan ${ }^{3}$, Miriam Wiley ${ }^{4}$, Aoifa Brick ${ }^{4}$, \\ Erik Clarke $^{5}$, Eadhbhard Mulligan ${ }^{1}$ \\ 1. Department of Surgery, Connolly Hospital Blanchardstown, Dublin, Ireland. 2. Faculty of Nursing \& Midwifery, Royal \\ College of Surgeons, Ireland. 3. Department of Surgical Affairs, Royal College of Surgeons, Ireland. 4. Department of \\ Health Research and Information, Economic and Social Research Institute, Dublin, Ireland. 5. Department of Health \\ Informatics, Royal College of Surgeons, Ireland.
}

Correspondence: Babak Meshkat, Specialist Registrar, General Surgery. Address: Connolly Hospital, Blanchardstown, Dublin 15, Ireland. E-mail: b_meshkat@hotmail.com

Received: July 15, 2013

DOI : $10.5430 /$ jha.v3n4p1
Accepted: October 9, 2013

URL: http://dx.doi.org/10.5430/jha.v3n4p1

\section{Abstract}

Background: The benefits of day surgery are supported internationally by the provision of standards. However, standards from one health jurisdiction are not readily transferable to others as national health strategy, policy and funding are influencing factors.

Objective: To determine, through consensus from experts in day surgery, a list of best practice statements for day surgery in Ireland.

Methods: A three round e-Delphi technique. Professionals in surgery, anaesthesia, nursing and management involved in day surgery across all hospitals in Ireland were invited to participate as the expert panel. In round $1 \mathrm{a}$ list of proposals for best practice were obtained from panel members. In round 2 experts were asked to rank each statement according to their importance on a nine point scale ( 1 = not important, 9 = high importance) using an online questionnaire. Consensus was set at $70 \%$, meaning the items that $70 \%$ of people deemed to be important were carried over to round 3 . A repeat online questionnaire was conducted with the remaining statements in round 3.

Results: Round 1 provided 261 statements. These were grouped and reduced to 62 statements for ranking. Following the iterative process over the subsequent two rounds a final list of 40 statements were developed and grouped into six thematic areas.

Conclusion: By using an e-Delphi process of gaining consensus among experts working in day surgical services, a list of best practice statements were developed.

\section{Key words}

Ambulatory surgery, Guidelines development, Delphi process 


\section{Introduction}

Internationally, reconfiguration of healthcare systems has focused attention on improving efficiency and cost effectiveness while simultaneously improving patient outcomes. Advances in anaesthesia and surgical techniques, contribute towards this goal as a greater number and range of surgical procedures can now be performed as day surgery, the benefits of which have been well documented ${ }^{[1]}$. The provision of standards for day surgery support this development. However, standards from one health jurisdiction are not readily transferable to other settings as national health strategy and policy are influencing factors. In Ireland there is currently a strategic national focus on surgical care and enhancement of day surgery is a particular objective. To achieve the goals of increasing day surgery rates it is necessary to engage people working in these services in the process of developing standards for day surgery which are appropriate in the Irish healthcare system and take cognisance of established international best practice.

A survey across public and private hospitals in Ireland, identified seven barriers to development and optimisation of day surgery ${ }^{[2]}$. Among these was the lack of nationally agreed standards. The purpose of this current study is to develop consensus based statements of best practice for day surgery which are specific, measurable, timely and appropriate to the Irish healthcare setting using a novel e-Delphi technique.

\section{Method}

The Delphi technique provides a method of obtaining consensus from individuals about issues where there is little or no definitive evidence and where opinion is important. The classical Delphi technique seeks to obtain group opinion through an anonymous, multilevel group interaction, relying on a number of rounds supervised by a facilitator to reach consensus ${ }^{[3]}$. The anonymity aspect avoids problems arising from powerful personalities, group pressure and the effects of status which often manifest more in the hierarchical structure of the health profession ${ }^{[4]}$. Variations on the classic Delphi have been reported including the use of on-line communication in the e-Delphi and more recently the development of the Hybrid Delphi ${ }^{[5]}$. The main characteristics of the Delphi are that it allows the anonymous inclusion of a large number of individuals across diverse locations and expertise and avoids dominance by any one individual ${ }^{[6]}$. It is an iterative process involving controlled feedback and statistical group response. The number of "rounds" or iterations is agreed at the start of the study and usually ranges from two to four. As our study aimed to develop national statements of best practice we sought to include nurses, doctors, anaesthetists and managers currently involved in day surgery who were diverse in terms of geographical location, professional affiliation and size of hospital unit. This group was deemed as experts on managing and conducting services for day surgery. Data collection was completed between November 2010 and January 2011 following Ethical approval by the Royal College of Surgeons in Ireland.

The level of consensus is usually set prior to the study and is influenced by the objectives of the study and the implications for practice ${ }^{[6,7]}$. We set a cut off level of $70 \%$ agreement on individual items relating to the range of best practice statements raised by respondents during rounds 2 and 3 of the e-Delphi.

There is no universally agreed format for round 1 in Delphi. Commensurate with the objectives of the study, multiple methods of recruitment of the expert panel was completed. Recruitment techniques for round 1 included a presentation of the proposed study at key scientific meetings for example a meeting of the British Association of Day Surgery (BADS) held in Dublin in November 2010. Focus groups were held at two national scientific surgical meetings; face to face interviews with surgeons and nurse managers were completed; a letter of invitation to each hospital providing day surgery in Ireland to propose a representative and to mailing lists which included directors of nursing, surgeons, surgical training, nurse managers and anaesthetists. Parallel to recruitment of participants a thorough search of databases and on-line resources was completed to identify other national standards or guidelines. Persons who agreed to study participation were requested to provide an email address through which all communications would take place. 
The study was completed using a commercially available online survey tool (http://www.surveymonkey.com). Security sockets layer (SSL) encryption is used by the service to protect data while being transmitted by ensuring a secure connection between a client (participant) and the server. This system offers the advantage of rapid response, instant analysis and supports the use of modern technology in gaining consensus among professionals who often have little time at their disposal. Having obtained the email addresses of respondents an email was issued to each study participant requesting them to open the survey monkey tool, answer the questions and press the submit button. In round 2, eDelphi participants were requested to address the following areas: patient information, pre-admission/pre-assessment, documentation, management of day surgery, and discharge protocols. Respondents were also requested to volunteer their email address for inclusion in subsequent rounds of e-Delphi.

Descriptive statistical analysis was completed using SPSS version 15. Agreement with statements was summarised using median scores and measures of dispersion.

For the open text box questions in round 1, qualitative data analysis was completed through thematic content analysis ${ }^{[8]}$. The list of items from round 1 was read by three team members working independently. Each member grouped similar themes together to develop a final refined list of statements. The team members compared the list of statements and similarities were noted with the final lists. Some differences were resolved through group discussion.

\subsection{Round one}

For round 1, each person was invited to propose items which they considered should be included in a list of statements of best practice for day surgery. A total of 54 email addresses were recorded. Of note, some of these were representative of groups or day surgery units so that the number of responses were greater than the number of email addresses.

\subsection{Round two}

The list of statements from round 1 was sent to each email address. Each participant was requested to state how important the item was on a nine point scale. With a nine point scale, scores 1-3 represents the region where participants feel the item is not indicated; 4-6 represent the region of equivocality; and 7-9 represent the region where it is felt that the item is indicated. Strict agreement is obtained if all rankings fall within one of these regions; a broad definition of agreement is obtained if ranks fall within any three-point regions. Once analysed, items for which $70 \%$ of participants did not rate within the scale of 7-9 were eliminated. These results were then fed back to participants for round 3.

\subsection{Round three}

In round 3, participants ranked the elements using the same nine point scale but this time with knowledge of the group scores. Thus, participants could reflect on their score and change with knowledge of the group score whilst also maintaining anonymity to the group. Similar to round 2, items which $70 \%$ of participants did not rank within the 7 groups - 9 groups were eliminated. Round 3 results were analysed in a similar manner to round 2 and a final list of statements agreed. This list was then further grouped into six major areas with each of the statements falling within one specific area. The figure demonstrates the steps of the e-Delphi process used in this study from recruitment until achievement of consensus.

\section{Results}

Over 500 personnel across all disciplines were informed about the study during the consultation process in round 1. Many verbally proposed items for the best practice through the face to face interviews and focus groups. Others forwarded comments and provided email addresses $(n=54)$ through the mailing lists. Some email addresses obtained were representative of groups, therefore the number of respondents was higher than the number of email addresses to which the survey was forwarded. There was a reduction in numbers participating in each round with the final round having 35 
participants (email addresses) of which 25.7\% $(n=9)$ were surgeons, 25.7\% $(n=9)$ anaesthetists, $37.1 \%(n=13)$ nurses, $5.7 \%(n=2)$ administrators and $5.7 \%(n=2)$ others.

A list of 62 statements was developed from round 1. This was then forwarded to participants to rate each item in round 2 (see the Figure).

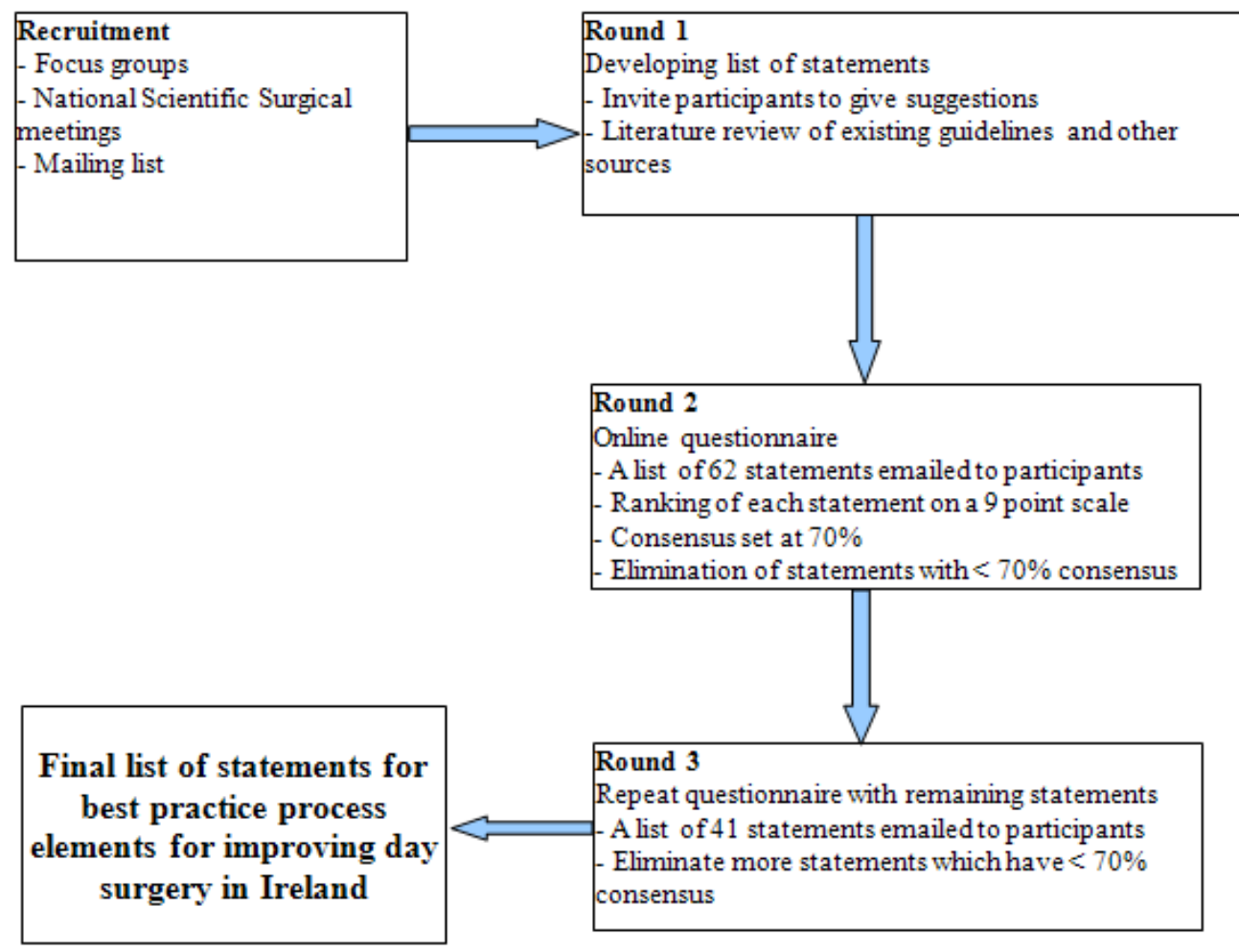

Figure. Step by step representation of the consensus process

Analysis of round 2 resulted in 21 statements being eliminated from further inclusion as they did not meet the $70 \%$ consensus criteria. The remaining 41 statements were returned to respondents for re-rating in round 3 . Analysis of round 3 resulted in only one further statement being eliminated, yielding forty statements.

The overall top five statements for best practice which were identified as very high importance by $97 \%(\mathrm{n}=34)$ of the expert panel were: 1) establishment of clear guidelines and protocols for admission to day surgery unit, 2) establishing patient selection criteria, 3) monitoring and audit of stay in rates, 4) provision of Information leaflets to give clear explanations of steps to be taken in the event of post discharge complications, and 5) ensuring adequate post operative pain relief prior to discharge.

The six headings under which each of the final forty statements were categorised were:

- Patient information

- Pre-admission/pre-assessment

- Documentation

- Management of day surgery

- Discharge protocols

- Monitoring of services 


\section{Discussion}

While there has been a rapid expansion of day surgery in Ireland in the past decade, recent studies suggest a lack of uniformity in service provision, leading to discrepancies in rates of day surgery achieved across hospitals that have similar proportion of day surgery beds and operating facilities ${ }^{[2]}$. Using the expertise and agreement of those working in day surgical services, a list of best practice statements for ensuring the provision of a uniformly safe and efficient day surgical services have been developed in this study. Although many of the statements used were similar existing guidelines and recommendations ${ }^{[9-14]}$ they have undergone a rigorous, stepwise consensus process to target the specific needs and challenges faced in the Irish healthcare setting.

\subsection{Patient information}

Patients undergoing day surgery are largely in charge of their own pre-operative preparation and post-operative recovery takes place at home. Such patients often have concerns which are different to those of patients undergoing surgery as in-patients. Patients undergoing day surgery often have concerns regarding whether they will be discharged too soon, if their condition deteriorates after they are discharged, would they receive adequate rest post operatively, whether they would becoming a burden on their family members, and managing postoperative complications (e.g. pain or postoperative nausea and vomiting (PONV) ${ }^{[15]}$. Therefore, it is essential for patients to not only receive adequate information in preparation for their surgery but also to be comfortable at the time of their discharge. The informing and education of patients is therefore essential to ensure cancellations and overnight admissions are minimised. This was reflected in the best practice statements developed in this study as the expert panel provided recommendations on requirements for pre-operative patient information and discharge information. Recommendations on pre-operative patient information included: information on the day surgery unit, procedure specific information for all patients, and information on the type of anaesthesia, all of which should be in the first language of the patient. Suggestions on discharge information included: advise on how to deal with common post-operative problems such as pain and emetic control, when to seek medical attention, what to do in case of emergency, as well as contact details for the unit or hospital.

\subsection{Pre-admission/ Pre-assessment}

In order to increase the frequency of day surgery and reduce the risk of adverse events, it is necessary to appropriately select patients and optimise those who are at a high risk ${ }^{[16]}$. Pre-assessment of patients provides this opportunity in a safe and efficient manner. In this study while the expert panel acknowledged the importance of pre-assessment and provided statements pertaining to requirements at pre-assessment, a clear distinction was made between pre-assessment and preanaesthetic assessment. The recommendations were that pre-assessment of patients should be a nurse led per protocol process. Where patients fail to meet the selection criteria for day surgery at pre-assessment, they should be referred to a pre-anaesthetic clinic conducted by a consultant anaesthetist where further assessment and optimisation can take place. The implications of this process is that patients that are immediately found suitable for day surgery at pre-assessment are put forward for day surgery, while those that do not meet the criteria at first assessment are investigated further and any necessary treatment initiated at pre-anaesthetic assessment. This process ensures that all patients are assessed for day surgery based on their individual physical status rather than arbitrarily selected based on age, body mass index (BMI) or American Society of Anaesthesiologists (ASA) score cut off points, thereby ensuring appropriate selection of patients to provide safe services to as many patients as possible and increasing overall day surgery rates.

\subsection{Documentation}

Good record keeping is a crucial part of safe and effective healthcare provision. Integrated care pathways provide a detailed, stepwise schema that cover the essential steps in the care of patients with a specific clinical problem ${ }^{[17]}$. The expert panel recommended that such integrated care pathways should be used in the day surgery setting in order for information to be easily accessible between the multidisciplinary team and the patients themselves. It was recommended that standardised integrated care pathway forms should be used for documentation from surgical out patients department 
(OPD) to pre-assessment, to day surgery and through to post discharge follow up. The recommendations further included that the care pathway forms should contain a consent form, which should be signed at the time of surgical OPD.

\subsection{Management of day surgery}

Strong leadership and management of day surgery units is a key factor to its success ${ }^{[1]}$. The implementation of policies that provide the advantages of day surgery to the patients, the healthcare professionals and the community at large is determined by those managing the service. In this study the expert panel provided several recommendations which could potentially improve day surgery rates. Of primary importance was providing dedicated day surgery theatre times. This would avoid late cancellation of day surgery cases, thereby increasing overall day surgery rates. Furthermore by developing working time tables which take into account peaks and troughs in emergency department activity levels, and where available extending day ward opening hours from 8:00 AM to 8:00 PM will further reduce overnight admission rates and late cancellations. It was also part of the expert's recommendations that a senior nurse should be in charge of the day surgery ward, and that patients should be contacted (telephone or text message) 48 hours prior to their appointment to remind them and ensure their attendance.

Some of these recommendations are already in practice in various hospitals across the country, however others may require changes such as the reorganisation of staffing in order for them to work effectively. This highlights the overall role of the statements of best practice that were developed in this study as they provide many criteria that should be set as targets for day surgery units to strive towards. As such they should be regularly evaluated and updated.

\subsection{Discharge protocols}

Protocols provide guidance and ensure uniformity of care for patients across different healthcare services. Having in place predetermined discharge criteria helps avoid unnecessary overnight admissions. The recommendations of the expert panel in this study highlighted the importance of adequate pain and emetic control as well as providing patients with information in case of emergency. Although currently very few hospitals follow up patients once they are discharged ${ }^{[2]}$, it was the recommendation of the panel that patients should be contacted within 24 hours post discharge to ensure adequate progress. This should be done through a telephone call by specially trained nursing staff who are familiar with the procedures, common post-operative complications and their presentation. Doing so provides assurance that patients will be followed up in the short post-operative period at home, and if any problems are encountered written protocols should be in place for actions to be taken. While some patients will have to be brought back to hospital, others may require further assessment in the community. It is therefore important as part of the discharge protocols that a link is also made between community based care and the day surgery unit so that in cases where the need arises, patients can be directly referred back to the hospital without having to go through the emergency department.

\subsection{Monitoring of services}

Clinical indicators are quantitative measurements related to one or more dimensions of performance and can be used to monitor, evaluate and improve the quality of patient care ${ }^{[1]}$. It is important that clinical indicators are both valid and reliable. As such, the panel of experts agreed that monitoring of stay in rates, adverse events, readmissions, cancellation rates and patient satisfaction surveys when taken together, would provide an overall assessment of quality and provision of services.

While monitoring of service provision to increase efficiency was an important part of the recommendations, it was also recognised that patient satisfaction is an equally important part of service provision. It would not be desirable to improve efficiency at the cost of patient satisfaction. The recommendation of the expert panel therefore placed particular emphasis on conduction of patient satisfaction surveys. Any intervention taken to improve service efficiency should be evaluated not to adversely impact on patient satisfaction. 


\subsection{E-Delphi as a method of achieving consensus}

There is limited reporting of the use of e-Delphi in the literature. This study found the e-Delphi process and online surveys to be economic in terms of investigator time and funding and facilitated rapid communication between the experts from different geographical locations and the research team thereby making it possible to reach a consensus in a timely fashion.

The falloff in response rate over the rounds of Delphi is a shortfall, however the process is longitudinal and is building consensus over time. Hsu Chia-Chien (2007) ${ }^{[3]}$ suggested that this shortfall in loosing participants is thought to be due to the fact that participants are asked to rate the same statements multiple times and can therefore lose interest. Despite the fall in response rate and changes in the types of experts participating in each round, the final results showed a wide range of areas where consensus was achieved.

Implementation of the final statements of best practice requires appropriate training of staff and in many cases reorganisation of day surgical services. Making changes in healthcare settings is often a difficult process especially when multiple disciplines are involved ${ }^{[18]}$. However going through the rigorous process of gaining consensus among the multidisciplinary teams that provide day surgery ensures that the recommendations are relevant and welcome by those who will eventually be implementing them. While this study outlines how consensus was achieved in developing statements of best practice for day surgery among experts, the effect of implementation of these remains to be elucidated. The project will now move on to the implantation phase of introducing the best practice statement in to a clinical setting.

\subsection{Role of statements of best practice}

The role of statements of best practice is to provide a baseline guide on set-up and operation of day surgery services which can apply to all day surgery providers in the Republic of Ireland. By going through the rigorous process of determining current existing barriers and gaining consensus among those working in day surgery services across the country on what are the statements of best practice, it is ensured that the process is transparent to those who will ultimately implement them and validated by the same professionals. It has been suggested that frequently the lack of the impact from new guidelines and standards is due to clashes in normative assumptions between the guidelines and their users ${ }^{[19]}$. While such clashes may never be completely avoided, the consensus method employed in this study facilitated an open discussion among professionals which would help ensure the statements are valid and accepted by those providing the services.

\section{Conclusion}

By using an e-Delphi process of gaining consensus among experts working in day surgical services in Ireland, a list of best practice statements were developed. The statements cover the entire patient journey from first presentation and assessment of suitability for day surgery to post-operative discharge protocols and follow-up. This is the first attempt at a national level to develop these statements, the majority of which share similarities with international standards ${ }^{[9-17]}$, which focus on initial appropriate patient selection through patient education and pre-assessment, clear documentation at every step of the patient journey, appropriate management of the unit and protocols in place to ensure post-operative management and discharge. The next challenge is to support implementation of these statements in order to optimise the provision of day surgery across Ireland.

\section{Conflict of interests}

The authors of this paper declare that they have no conflict of interests.

\section{References}

[1] Castoro C, Bertinato L, Baccaglini U, Drace CA, McKee M. Policy Brief, Day Surgery: Making it happen, European Observatory on Healthsystems and Policies. 2007. 
[2] Cowman S, Gethin G, Mulligan E, Ryan K, Meshkat B. National survey of the provision of day surgery across public and private hospitals in Ireland. Ir J Med Sci. 2010 Dec; 179(4): 493-9. Epub 2010 Aug 29. PMID: 20803318. http://dx.doi.org/10.1007/s11845-010-0562-7

[3] Hsu Chia-Chien. The Delphi Technique: Making Sense of Consensus, Practical Assessment Research and Evaluation, 2007; volume 12, number 10. ISSN 1531-7714.

[4] Williams PL, Webb C. The Delphi technique: a methodological discussion J Adv Nurs. 1994 Jan; 19(1): $180-6$. PMID: 8138622. http://dx.doi.org/10.1111/j.1365-2648.1994.tb01066.x

[5] Landeta J, Barrutia J, Lertxundi A. Hybrid Delphi: A methodology to facilitate contributions from experts in professional contexts. Technol Forecast Soc Change, April 2011. online publication. http://dx.doi.org/10.1016/j.techfore.2011.03.009

[6] Keeney S, Hasson F, McKenna H. Consulting the oracle: ten lessons from using the Delphi technique in nursing research. J Adv Nurs. 2006 Jan; 53(2): 205-12. PMID: 16422719. http://dx.doi.org/10.1111/j.1365-2648.2006.03716.x

[7] Hasson F, Keeney S, McKenna H. Research guidelines for the Delphi survey technique. J Adv Nurs. 2000 Oct; 32 (4): $1008-15$. PMID: 11095242.

[8] Colaizzi PP. Psychological research as the phenomenologist views it. In Existential Phenomenological Alternatives to Psychology (Valle RS \& King M eds). 1978. Oxford University, New York. Page: 48-71.

[9] Day Surgery in Australia, Report and Recommendations of the Australian Day Surgery Council of Royal Australian College of Surgeons, Australian and New Zeland College of Anaesthetists and The Australian Society of the Anaesthetists, Revised edition. 2004.

[10] Department of Health. Day surgery: Operational guide, Waiting, booking and choice. August 2002.

[11] NHS Modernisation Agency. National good practice guidance on pre-operative assessment for day surgery, Department of Health, London. 2002.

[12] The Association of Anaesthetists of Great Britain and Ireland. Pre-operative Assessment The Role of the Anaesthetist. Nov 2001.

[13] Welsh audit office. Making better use of NHS day surgery in Wales. Sept 2006.

[14] Ministry of health and consumer affairs in Spain. Day surgery unit guide: standards and recommendations. 2008.

[15] Marley RA, Swanson J. Patient care after discharge from the ambulatory surgical center, J Perianesth Nurs. 2001 Dec; 16(6): 399-417; quiz 417-9. PMID: 11740781. http://dx.doi.org/10.1053/jpan.2001.28891

[16] Bettelli G. High risk patients in day surgery, Minerva Anestesiol. 2009 May; 75(5): 259-68. Epub 2008 Oct 23. PMID: 18946427.

[17] Campbell H, Hotchkiss R, Bradshaw N, Porteous M. Integrated care pathways, BMJ. 1998 Jan 10; 316(7125): 133-7. PMID: 9462322. http://dx.doi.org/10.1136/bmj.316.7125.133

[18] NHS CENTRE FOR REVIEWS \& DISSEMINATION. Getting Evidence Into Practice. The Royal Society of Medicine Press Limited., York: University of York., February 1999. Volume 5, Number 1, ISSN 0965-0288.

[19] Royal Society of Medicine Press Limited, York: University of York. February 1999; Volume 5, Number 1, ISSN $0965-0288$. 